\title{
FRACTIONAL AND INTEGER MATCHINGS IN UNIFORM HYPERGRAPHS
}

\author{
DANIELA KÜHN, DERYK OSTHUS AND TIMOTHY TOWNSEND
}

\begin{abstract}
A conjecture of Erdős from 1965 suggests the minimum number of edges in a $k$ uniform hypergraph on $n$ vertices which forces a matching of size $t$, where $t \leq n / k$. Our main result verifies this conjecture asymptotically, for all $t<0.48 n / k$. This gives an approximate answer to a question of Huang, Loh and Sudakov, who proved the conjecture for $t \leq n / 3 k^{2}$. As a consequence of our result, we extend bounds of Bollobás, Daykin and Erdős by asymptotically determining the minimum vertex degree which forces a matching of size $t<0.48 n /(k-1)$ in a $k$-uniform hypergraph on $n$ vertices. We also obtain further results on $d$-degrees which force large matchings. In addition we improve bounds of Markström and Ruciński on the minimum $d$ degree which forces a perfect matching in a $k$-uniform hypergraph on $n$ vertices. Our approach is to inductively prove fractional versions of the above results and then translate these into integer versions.
\end{abstract}

\section{INTRODUCTION}

1.1. Large matchings in hypergraphs with many edges. A $k$-uniform hypergraph is a pair $G=(V, E)$ where $V$ is a finite set of vertices and the edge set $E$ consists of unordered $k$-tuples of elements of $V$. A matching (or integer matching) $M$ in $G$ is a set of disjoint edges of $G$. The size of $M$ is the number of edges in $M . M$ is perfect if it has size $|V| / k$.

A classical theorem of Erdős and Gallai [9] determines the number of edges in a graph which forces a matching of a given size. In 1965, Erdős [8] made a conjecture which would generalize this to $k$-uniform hypergraphs.

Conjecture 1.1. Let $n, k \geq 2$ and $1 \leq s \leq n / k$ be integers. The minimum number of edges in a $k$-uniform hypergraph on $n$ vertices which forces a matching of size $s$ is

$$
\max \left\{\left(\begin{array}{c}
k s-1 \\
k
\end{array}\right),\left(\begin{array}{l}
n \\
k
\end{array}\right)-\left(\begin{array}{c}
n-s+1 \\
k
\end{array}\right)\right\}+1
$$

It is easy to see that the conjecture would be best possible: the first expression in the lower bound is obtained by considering the $k$-uniform clique $K_{k s-1}^{(k)}$ (complemented by $n-k s+1$ isolated vertices); the second expression in the lower bound is obtained as follows. Let $H(s)$ be a $k$-uniform hypergraph on $n$ vertices with edge set consisting of all $k$-element subsets of $V(H(s))$ intersecting a given subset of $V(H(s))$ of size $s-1$, that is $H(s)=K_{n}^{(k)}-K_{n-s+1}^{(k)}$.

The case $s=2$ of Conjecture 1.1 corresponds to the Erdős-Ko-Rado Theorem on intersecting families [10]. The conjecture also has applications to the Manickam-Mikós-Singhi conjecture in number theory (for details see e.g. [2]). Despite its seeming simplicity Conjecture 1.1 is still wide open in general. For the cases $k \leq 4$, it was verified asymptotically by Alon, Frankl, Huang, Rödl, Ruciński and Sudakov [1]. For $k=3$, it was recently proved by Frankl [11], improving

Date: April 24, 2013.

D. Kühn was supported by the ERC, grant no. 258345. D. Osthus was supported by the ERC, grant no. 306349. 
results of Frankl, Rödl and Ruciński [12], and of Łuczak and Mieczkowska [21]. Bollobás, Daykin and Erdős [4] proved Conjecture 1.1 for general $k$ whenever $s<n /\left(2 k^{3}\right)$, which extended earlier results of Erdős [8]. Huang, Loh and Sudakov [14] proved it for $s<n /\left(3 k^{2}\right)$. The main result in this paper verifies Conjecture 1.1 asymptotically for matchings of any size up to almost half the size of a perfect matching. This gives an asymptotic answer to a question in [14].

Theorem 1.2. Let $n, k \geq 2$ and $0 \leq a<0.48 / k$ be such that $n, k$, an $\in \mathbb{N}$. The minimum number of edges in a $k$-uniform hypergraph on $n$ vertices which forces a matching of size an is

$$
\left(1-(1-a)^{k}+o(1)\right)\left(\begin{array}{l}
n \\
k
\end{array}\right)
$$

1.2. Large matchings in hypergraphs with large degrees. It is also natural to consider degree conditions that force matchings in uniform hypergraphs. Given a $k$-uniform hypergraph $G=(V, E)$ and $S \in\left(\begin{array}{l}V \\ d\end{array}\right)$, where $0 \leq d \leq k-1$, let $\operatorname{deg}_{G}(S)=|\{e \in E: S \subseteq e\}|$ be the degree of $S$ in $G$. Let $\delta_{d}(G)=\min _{S \in\left(\begin{array}{c}V \\ d\end{array}\right)}\left\{\operatorname{deg}_{G}(S)\right\}$ be the minimum $d$-degree of $G$. When $d=1$, we refer to $\delta_{1}(G)$ as the minimum vertex degree of $G$. Note that $\delta_{0}(G)=|E|$.

For integers $n, k, d, s$ satisfying $0 \leq d \leq k-1$ and $0 \leq s \leq n / k$, we let $m_{d}^{s}(k, n)$ denote the minimum integer $m$ such that every $k$-uniform hypergraph $G$ on $n$ vertices with $\delta_{d}(G) \geq m$ has a matching of size $s$. So the results discussed in Section 1.1 correspond to the case $d=0$. The following degree condition for forcing perfect matchings has been conjectured in $[13,18]$ and also received much attention recently.

Conjecture 1.3. Let $n$ and $1 \leq d \leq k-1$ be such that $n, d, k, n / k \in \mathbb{N}$. Then

$$
m_{d}^{n / k}(k, n)=\left(\max \left\{\frac{1}{2}, 1-\left(\frac{k-1}{k}\right)^{k-d}\right\}+o(1)\right)\left(\begin{array}{l}
n-d \\
k-d
\end{array}\right) .
$$

The lower bound here is given by the hypergraph $H(n / k)$ defined in Section 1.1 and the following parity-based construction from [17]. For any integers $n, k$, let $H^{\prime}$ be a $k$-uniform hypergraph on $n$ vertices with vertex partition $A \cup B=V\left(H^{\prime}\right)$, such that $\| A|-| B|| \leq 2$ and $|A|$ and $n / k$ have different parity. Let $H^{\prime}$ have edge set consisting of all $k$-element subsets of $V\left(H^{\prime}\right)$ that intersect $A$ in an odd number of vertices. Observe that $H^{\prime}$ has no perfect matching, and that for every $1 \leq d \leq k-1$ we have that $\delta_{d}\left(H^{\prime}\right)=(1 / 2+o(1))\left(\begin{array}{l}n-d \\ k-d\end{array}\right)$.

For $d=k-1, m_{k-1}^{n / k}(k, n)$ was determined exactly by Rödl, Ruciński and Szemerédi [29]. This was generalized by Treglown and Zhao [32], who determined the extremal families for all $d \geq k / 2$. The extremal constructions are similar to the parity based one of $H^{\prime}$ above. This improves asymptotic bounds in [25, 28, 29]. For $d<k / 2$ less is known. In [1] Conjecture 1.3 was proved for $k-4 \leq d \leq k-1$, by reducing it to a probabilistic conjecture of Samuels. In particular, this implies Conjecture 1.3 for $k \leq 5$. Khan [15], and independently Kühn, Osthus and Treglown [19], determined $m_{1}^{n / k}(k, n)$ exactly for $k=3$. Khan [16] also determined $m_{1}^{n / k}(k, n)$ exactly for $k=4$. As a consequence of these results, $m_{1}^{s}(k, n)$ is determined exactly whenever $s \leq n / k$ and $k \leq 4$ (for details see the concluding remarks in [19]). More generally, we propose the following version of Conjecture 1.3 for non-perfect matchings.

Conjecture 1.4. For all $\varepsilon>0$ and all integers $n, d, k, s$ with $1 \leq d \leq k-1$ and $0 \leq s \leq$ $(1-\varepsilon) n / k$ we have

$$
m_{d}^{s}(k, n)=\left(1-\left(1-\frac{s}{n}\right)^{k-d}+o(1)\right)\left(\begin{array}{l}
n-d \\
k-d
\end{array}\right)
$$


In fact it may be that the bound holds for all $s \leq n-C$, for some $C$ depending only on $d$ and $k$. The lower bound here is given by $H(s)$. The case $d=k-1$ of Conjecture 1.4 follows easily from the determination of $m_{k-1}^{s}(k, n)$ for $s$ close to $n / k$ in [29]. Bollobás, Daykin and Erdős [4] determined $m_{1}^{s}(k, n)$ for small $s$, i.e. whenever $s<n / 2 k^{3}$. As a consequence of our main result, for $1 \leq d \leq k-2$ we are able to determine $m_{d}^{s}(k, n)$ asymptotically for non-perfect matchings of any size less than $0.48 n /(k-d)$. Note that this proves Conjecture 1.4 in the case $0.53 k \leq d \leq k-2$, say.

Theorem 1.5. Let $\varepsilon>0$ and let $n, k, d$ be integers with $1 \leq d \leq k-2$, and let $0 \leq a<$ $\min \{0.48 /(k-d),(1-\varepsilon) / k\}$ be such that an $\in \mathbb{N}$. Then

$$
m_{d}^{a n}(k, n)=\left(1-(1-a)^{k-d}+o(1)\right)\left(\begin{array}{l}
n-d \\
k-d
\end{array}\right) .
$$

We now focus again on the case $s=n / k$, i.e. perfect matchings. It was shown by Hàn, Person and Schacht [13] that for $k \geq 3,1 \leq d<k / 2$ we have $m_{d}^{n / k}(k, n) \leq((k-d) / k+o(1))\left(\begin{array}{l}n-d \\ k-d\end{array}\right)$. (The case $d=1$ of this is already due to Daykin and Häggkvist [6].) These bounds were slightly improved by Markström and Ruciński [22], using similar techniques, to

$$
m_{d}^{n / k}(k, n) \leq\left(\frac{k-d}{k}-\frac{1}{k^{k-d}}+o(1)\right)\left(\begin{array}{l}
n-d \\
k-d
\end{array}\right) .
$$

Using similar methods to those developed to prove Theorem 1.5, we are also able to slightly improve on this bound.

Theorem 1.6. Let $n$ and $1 \leq d<k / 2$ be such that $n, k, d, n / k \in \mathbb{N}$. Then

$$
m_{d}^{n / k}(k, n) \leq\left(\frac{k-d}{k}-\frac{k-d-1}{k^{k-d}}+o(1)\right)\left(\begin{array}{l}
n-d \\
k-d
\end{array}\right) .
$$

1.3. Large fractional matchings. Our approach to proving these results uses the concepts of fractional matchings and fractional vertex covers. A fractional matching in a $k$-uniform hypergraph $G=(V, E)$ is a function $w: E \rightarrow[0,1]$ of weights of edges, such that for each $v \in V$ we have $\sum_{e \in E: v \in e} w(e) \leq 1$. The size of $w$ is $\sum_{e \in E} w(e) . w$ is perfect if it has size $|V| / k$. A fractional vertex cover in $G$ is a function $w: V \rightarrow[0,1]$ of weights of vertices, such that for each $e \in E$ we have $\sum_{v \in e} w(v) \geq 1$. The size of $w$ is $\sum_{v \in V} w(v)$.

A key idea (already used e.g. in $[1,28]$ ) is that we can switch between considering the largest fractional matching and the smallest fractional vertex cover of a hypergraph. The determination of these quantities are dual linear programming problems, and hence by the Duality Theorem they have the same size.

For $s \in \mathbb{R}$ we let $f_{d}^{s}(k, n)$ denote the minimum integer $m$ such that every $k$-uniform hypergraph $G$ on $n$ vertices with $\delta_{d}(G) \geq m$ has a fractional matching of size $s$. It was shown in [28] that $f_{k-1}^{n / k}(k, n)=\lceil n / k\rceil$. Similarly to [1], we now formulate the fractional version of Conjecture 1.1.

Conjecture 1.7. For all integers $n, k, s$ with $k \geq 2$ and $1 \leq s \leq n / k$ we have

$$
f_{0}^{s}(k, n)=\max \left\{\left(\begin{array}{c}
k s-1 \\
k
\end{array}\right),\left(\begin{array}{l}
n \\
k
\end{array}\right)-\left(\begin{array}{c}
n-s+1 \\
k
\end{array}\right)\right\}+1 .
$$

As discussed in [1], this conjecture has applications to a problem on information storage and retrieval. To prove Theorems 1.2 and 1.5, we first prove Conjecture 1.7 asymptotically for fractional matchings of any size up to $0.48 n / k$. 
Theorem 1.8. Let $n, k \geq 2$ be integers and let $0 \leq a \leq 0.48 / k$. Then

$$
f_{0}^{a n}(k, n)=\left(1-(1-a)^{k}+o(1)\right)\left(\begin{array}{l}
n \\
k
\end{array}\right) .
$$

We use Theorem 1.8, along with methods similar to those developed in [1], to convert our edge-density conditions for the existence of fractional matchings into corresponding minimum degree conditions (see Proposition 4.1). For $1 \leq d \leq k-2$ the following theorem asymptotically determines $f_{d}^{s}(k, n)$ for fractional matchings of any size up to $0.48 n /(k-d)$. Note that this determines $f_{d}^{s}(k, n)$ asymptotically for all $s \in(0, n / k)$ whenever $d \geq 0.52 k$.

Theorem 1.9. Let $n, k \geq 3$, and $1 \leq d \leq k-2$ be integers and let $0 \leq a \leq \min \{0.48 /(k-$ d), $1 / k\}$. Then

$$
f_{d}^{a n}(k, n)=\left(1-(1-a)^{k-d}+o(1)\right)\left(\begin{array}{l}
n-d \\
k-d
\end{array}\right)
$$

We then use Theorem 1.8 and a variant of Theorem 1.9, along with the Weak Hypergraph Regularity Lemma, to prove Theorems 1.2 and 1.5 respectively, by converting our fractional matchings into integer ones. We prove Theorem 1.6 in a similar fashion, via the following two theorems.

Theorem 1.10. Let $n, k \geq 2, d \geq 1$ be integers. Then

$$
f_{0}^{n /(k+d)}(k, n) \leq\left(\frac{k}{k+d}-\frac{k-1}{(k+d)^{k}}+o(1)\right)\left(\begin{array}{l}
n \\
k
\end{array}\right) .
$$

Theorem 1.11. Let $n, k \geq 3,1 \leq d \leq k-2$ be integers. Then

$$
f_{d}^{n / k}(k, n) \leq\left(\frac{k-d}{k}-\frac{k-d-1}{k^{k-d}}+o(1)\right)\left(\begin{array}{l}
n-d \\
k-d
\end{array}\right)
$$

The rest of the paper is organised as follows. In Section 2 we lay out some notation, set out some useful tools, and prove some preliminary results. Section 3 is the heart of the paper, in which we prove Theorems 1.8 and 1.10. In Section 4 we derive Theorems 1.9 and 1.11, and in Section 5 we derive Theorems 1.2, 1.5 and 1.6. In Section 6 we discuss an application of our main result to the problem of determining the minimum number of disjoint cliques in graphs of given edge density.

\section{Notation, tOols AND PRELIMINARY RESUlts}

2.1. Notation. As in many of the proofs in this paper we often consider vertex degrees, we may write $\delta(G)$ to denote $\delta_{1}(G)$. Similarly, when $S=\{v\}$ is a set containing only one vertex, we write $d_{G}(v)$ to denote $\operatorname{deg}_{G}(S)$, and we refer to $d_{G}(v)$ as the degree of $v$ (in $G$ ). We let $e(G)$ denote the number of edges in a hypergraph $G$, and let $|G|$ denote the number of its vertices. For a set $V$ and a positive integer $k$ we let $\left(\begin{array}{l}V \\ k\end{array}\right)$ denote the set of all $k$-element subsets of $V$. For $m \in \mathbb{N}$ we let $[m]$ denote the set $\{1, \ldots, m\}$. Whenever we refer to a $k$-tuple, we assume that it is unordered. Given a hypergraph $G=(V, E)$ and a set $S \subseteq V$, we refer to the pair $(V \backslash S,\{e \subseteq V: S \cap e=\emptyset, e \cup S \in E\})$ as the neighbourhood hypergraph of $S$ (in $G$ ). If $S=\{v\}$ has just one element then we may refer to this pair as the neighbourhood hypergraph of $v$. For $U \subseteq V$ we denote by $G[U]$ the hypergraph induced by $U$ on $G$, that is the hypergraph with vertex set $U$ and edge set $\{e \in E: e \subseteq U\}$. 
2.2. Fractional matchings and duality. In proving our main theorems, we will use the lower bound given by the earlier construction $H(s)$, for all integers $n, d, k, s$ with $k \geq 2$ and $0 \leq d \leq k-1$ and $0 \leq s \leq n / k$ :

$$
m_{d}^{s}(k, n) \geq f_{d}^{s}(k, n) \geq\left(1-(1-s / n)^{k-d}+o(1)\right)\left(\begin{array}{l}
n-d \\
k-d
\end{array}\right) .
$$

Now, as mentioned in Section 1, a key tool in this paper is that the determination of the size of the largest fractional matching of a $k$-uniform hypergraph is a linear programming problem, and its dual problem is to determine the size of the smallest fractional vertex cover of the hypergraph. The following proposition, which follows by the Duality Theorem, will be very useful to us.

Proposition 2.1. Let $k \geq 2$ and let $G$ be a k-uniform hypergraph. The size of the largest fractional matching of $G$ is equal to the size of the smallest fractional vertex cover of $G$.

In the rest of this section we collect some preliminary results.

Proposition 2.2. Let $G=(V, E)$ be a hypergraph, $E^{\prime} \subseteq E, S \subseteq V$, and let $w$ be a fractional vertex cover of $G$. Then

$$
e(G) \leq \sum_{e \in E} \sum_{v \in e \backslash S} w(v)+\sum_{e \in E^{\prime}} \sum_{v \in e \cap S} w(v)+\left|E \backslash E^{\prime}\right| .
$$

Proof. As $w$ is a fractional vertex cover of $G$,

$$
e(G)=\left|E^{\prime}\right|+\left|E \backslash E^{\prime}\right| \leq \sum_{e \in E^{\prime}} \sum_{v \in e} w(v)+\left|E \backslash E^{\prime}\right| \leq \sum_{e \in E} \sum_{v \in e \backslash S} w(v)+\sum_{e \in E^{\prime}} \sum_{v \in e \cap S} w(v)+\left|E \backslash E^{\prime}\right| .
$$

The following crude bound will sometimes be useful. The proof is immediate from the definitions.

Proposition 2.3. Suppose that $k \geq 2$ and $0<a, c<1$ are fixed. Then for every $\varepsilon>0$ there exists $n_{0}=n_{0}(k, \varepsilon)$ such that if $n \geq n_{0}$ and $f_{0}^{a n}(k, n) \leq c\left(\begin{array}{l}n \\ k\end{array}\right)$ then $f_{0}^{a n+1}(k, n) \leq(c+\varepsilon)\left(\begin{array}{l}n \\ k\end{array}\right)$.

In the next section we will prove Theorem 1.8 by induction. For this we will need Theorem 2.4, which will establish the base case of this induction. Theorem 2.4 is an easy consequence of the Erdős-Gallai Theorem from [9].

Theorem 2.4. For $k=2$ and $x \leq 1 / 3$ we have

$$
f_{0}^{x n}(k, n)=\left(1-(1-x)^{k}+o(1)\right)\left(\begin{array}{l}
n \\
k
\end{array}\right) .
$$

2.3. Numerical estimates. We now define a function that will appear a number of times throughout this paper. For $k \geq 3$, let

$$
g_{k}(x):=\frac{1-(1-2 x)^{k-1}}{(1-x)^{k-1}} .
$$

Proposition 2.5. Let $k \in \mathbb{N}$ with $k \geq 3$. The equation $g_{k}(x)=1$ has a unique solution in $(0,1 /(k+1))$. 
Proof. Note that $g_{k}(x)$ is strictly increasing for $x \in(0,1 /(k+1))$ and that $g_{k}(0)=0$. Also note that

$$
\begin{aligned}
g_{k}(x) & =\frac{1-(1-2 x)^{k-1}}{(1-x)^{k-1}} \frac{(1+x)^{k-1}}{(1+x)^{k-1}}=\frac{(1+x)^{k-1}-\left(1-x-2 x^{2}\right)^{k-1}}{\left(1-x^{2}\right)^{k-1}} \\
& >\frac{(1+x)^{k-1}-(1-x)^{k-1}}{\left(1-x^{2}\right)^{k-1}}>(1+x)^{k-1}-(1-x)^{k-1}>2(k-1) x,
\end{aligned}
$$

for $x \in(0,1 / 2)$, so $g_{k}(1 /(k+1))>2(k-1) /(k+1) \geq 1$ for $k \geq 3$. So indeed $g_{k}(x)=1$ has a unique solution in $(0,1 /(k+1))$.

We define $a_{k}^{\prime}$ to be the unique solution in $(0,1 /(k+1))$ to $g_{k}(x)=1$.

Proposition 2.6. Let $k \geq 3$. Then $a_{k}^{\prime}>0.48 / k$.

The proof of Theorem 1.8 actually extends to all $a<a_{k}^{\prime}$ (rather than just $a \leq 0.48 / k$ ). For small $k$ this gives a substantial improvement (at the expense of lengthy calculations). But for large $k$, a similar argument to that in the proof of Proposition 2.6 shows that $a_{k}^{\prime} \leq 0.49 / k$, so even improving the bound to $a \leq 1 / 2 k$ say would require new ideas.

Proof. Let $x \in(0,1 /(k+1))$. Note that, as $e^{-u-u^{2}} \leq 1-u$ for all $u \in(0,1 / 2)$, we have that

$$
\begin{aligned}
g_{k}(x) & =\frac{1-(1-2 x)^{k-1}}{(1-x)^{k-1}} \leq \frac{1-e^{-2 x(k-1)-4 x^{2}(k-1)}}{e^{-x(k-1)-x^{2}(k-1)}}=e^{x^{2}(k-1)-x}\left(e^{x k}-e^{-x(k-2)-4 x^{2}(k-1)}\right) \\
& =e^{x^{2}(k-1)-x}\left(2 \sinh (x k)+e^{-x k}\left(1-e^{-4 x^{2}(k-1)+2 x}\right)\right) .
\end{aligned}
$$

So, as $0 \leq x \leq 1 / k$,

$$
g_{k}(x) \leq e^{1 / k}\left(2 \sinh (x k)+1-e^{-4 / k}\right) \leq e^{1 / k}\left(2 \sinh (x k)+\frac{4}{k}\right)=: f_{k}(x) .
$$

The last inequality holds as $e^{-u} \geq 1-u$ for all $u \in \mathbb{R}$. Now, $f_{2000}(0.48 / 2000)$ is approximately $0.99979<1$. Note that both $f_{k}(x)$ and $g_{k}(x)$ are strictly increasing for $x \in(0,1 /(k+1))$, and $f_{k}(0.48 / k)=e^{1 / k}(2 \sinh (0.48)+4 / k)$ is strictly decreasing in $k$, and $f_{k}(x) \geq g_{k}(x)$ for $x \in(0,1 /(k+1))$. It follows that $a_{k}^{\prime}>0.48 / k$ for all $k \geq 2000$. It can be easily verified that $a_{k}^{\prime}>0.48 / k$ for all $3 \leq k<2000$. Indeed, as $g_{k}(x)$ is strictly increasing for $x \in(0,1 /(k+1))$, it suffices to verify, e.g. using Maple, that $g_{k}(0.48 / k)<1$ for all $3 \leq k<2000$. This completes the proof.

The next proposition will be needed in the proof of Theorem 1.10. To prove this proposition we will need a well-known theorem of Baranyai [3] from 1975.

Theorem 2.7 (Baranyai's Theorem). If $n \in \ell \mathbb{N}$ then the complete $\ell$-uniform hypergraph on $n$ vertices decomposes into edge-disjoint perfect matchings.

Proposition 2.8. Let $n, k, \ell$ be integers with $k \geq 2$ and $1 \leq \ell \leq k$, and let $\eta \in[0,1)$. Let $V$ be a set of size $n$. Suppose $S \subseteq V$, with $|S| \in \ell \mathbb{N}$. Then there exists $\tilde{E} \subseteq\left\{e \in\left(\begin{array}{l}V \\ k\end{array}\right):|e \cap S|=\ell\right\}$ such that for every $v \in S$,

$$
|\{e \in \tilde{E}: v \in e\}|=\left\lfloor\eta\left(\begin{array}{c}
|S| \\
\ell-1
\end{array}\right)\left(\begin{array}{c}
n-|S| \\
k-\ell
\end{array}\right)\right\rfloor .
$$


Proof. The cases where $\ell=1$ or $\eta=0$ are trivial. So suppose that $2 \leq \ell \leq k$ and $\eta \in(0,1)$. Apply Theorem 2.7 to find a decomposition of the complete $\ell$-uniform hypergraph on $S$ into edge-disjoint perfect matchings $M_{1}, \ldots, M_{\left(\begin{array}{c}|S|-1 \\ \ell-1\end{array}\right)}$.

We now construct $\tilde{E}$ by adding $k$-tuples from $\left\{e \in\left(\begin{array}{l}V \\ k\end{array}\right):|e \cap S|=\ell\right\}$ greedily, under the following constraints:

(i) for all $i \in\left\{1, \ldots,\left(\begin{array}{c}|S|-1 \\ \ell-1\end{array}\right)\right\}$, we do not add any $k$-tuples in $\left\{e \in\left(\begin{array}{c}V \\ k\end{array}\right): e \cap S \in M_{i+1}\right\}$ unless we have already added all $k$-tuples in $\left\{e \in\left(\begin{array}{l}V \\ k\end{array}\right): e \cap S \in M_{i}\right\}$;

(ii) for every $v \in S$,

$$
|\{e \in \tilde{E}: v \in e\}| \leq \eta\left(\begin{array}{c}
|S| \\
\ell-1
\end{array}\right)\left(\begin{array}{c}
n-|S| \\
k-\ell
\end{array}\right) .
$$

It is clear that (i) and (ii) ensure that the set $\tilde{E}$ obtained in this way satisfies (2.2) for every $v \in S$.

\section{Minimum EdGe-Density COnditions For FRACTiOnal Matchings}

We will use the following lemma to prove Theorems 1.8 and 1.10 inductively.

Lemma 3.1. Let $k \geq 3$ be fixed. Suppose that $a \in(0,1 /(k+1)], c \in(0,1)$ and that there exists $n_{0} \in \mathbb{N}$ such that for all $n \geq n_{0}$ we have

$$
f_{0}^{a n /(1-a)}(k-1, n) \leq c\left(\begin{array}{c}
n \\
k-1
\end{array}\right) .
$$

Then for all $\varepsilon>0$ there exists $n_{1} \in \mathbb{N}$ such that for all $n \geq n_{1}$ any $k$-uniform hypergraph $G$ on $n$ vertices with at least an vertices of degree at least

$$
D:=\left(c(1-a)^{k-1}+\left(1-(1-a)^{k-1}\right)+\varepsilon\right)\left(\begin{array}{l}
n-1 \\
k-1
\end{array}\right)
$$

has a fractional matching of size an.

Proof. Let $\varepsilon>0$ and choose $n_{1}$ sufficiently large. Consider a $k$-uniform hypergraph $G=(V, E)$ on $n$ vertices, of which at least an have degree at least $D$. Let $Y \subseteq V$ be the set of $\lceil a n\rceil$ vertices of highest degree. Let $w$ be a fractional vertex cover of $G$ of least size. Consider the vertex $v_{0} \in Y$ with the lowest weight $w\left(v_{0}\right)$. Let $H$ be the neighbourhood hypergraph of $v_{0}$ in $G$. So

$$
e(H)=d_{G}\left(v_{0}\right) \geq D=\left(c(1-a)^{k-1}+\left(1-(1-a)^{k-1}\right)+\varepsilon\right)\left(\begin{array}{l}
n-1 \\
k-1
\end{array}\right) .
$$

Let $H^{\prime}:=H[V \backslash Y]$. Since the number of edges in $H$ with at least one vertex in $Y$ is at most $\left(1-(1-a)^{k-1}+o(1)\right)\left(\begin{array}{c}n-1 \\ k-1\end{array}\right)$, it follows that

$$
\begin{aligned}
e\left(H^{\prime}\right) & \geq e(H)-\left(1-(1-a)^{k-1}+o(1)\right)\left(\begin{array}{l}
n-1 \\
k-1
\end{array}\right) \geq\left(c(1-a)^{k-1}+\varepsilon / 2\right)\left(\begin{array}{l}
n-1 \\
k-1
\end{array}\right) \\
& \geq(c+\varepsilon / 3)\left(\begin{array}{c}
\left|H^{\prime}\right| \\
k-1
\end{array}\right),
\end{aligned}
$$

where in the last two inequalities we use that $n_{1}$ was chosen sufficiently large. Note that $\left|H^{\prime}\right| \geq n / 2$, so we may assume that $\left|H^{\prime}\right| \geq n_{0}$. Now, (3.1) and Proposition 2.3 together imply that $H^{\prime}$ has a fractional matching of size

$$
a\left|H^{\prime}\right| /(1-a)+1=a(n-\lceil a n\rceil) /(1-a)+1 \geq a n .
$$


So let $M$ be a fractional matching of $H^{\prime}$ of size an. Note that for all $v \in V \backslash Y$,

$$
\sum_{e \in E\left(H^{\prime}\right): v \in e} M(e) \leq 1 .
$$

So we have that

$$
\sum_{v \in V} w(v) \geq \sum_{v \in Y} w(v)+\sum_{e \in E\left(H^{\prime}\right)} \sum_{v \in e} M(e) w(v) .
$$

By the minimality of $w\left(v_{0}\right)$, this implies that

$$
\begin{aligned}
\sum_{v \in V} w(v) & \geq a n w\left(v_{0}\right)+\sum_{e \in E\left(H^{\prime}\right)} \sum_{v \in e} M(e) w(v)=\sum_{e \in E\left(H^{\prime}\right)} M(e) w\left(v_{0}\right)+\sum_{e \in E\left(H^{\prime}\right)} \sum_{v \in e} M(e) w(v) \\
& =\sum_{e \in E\left(H^{\prime}\right)} M(e)\left(w\left(v_{0}\right)+\sum_{v \in e} w(v)\right) \geq \sum_{e \in E\left(H^{\prime}\right)} M(e)=a n .
\end{aligned}
$$

The last inequality holds because by definition of $H^{\prime}$ we have $e \cup\left\{v_{0}\right\} \in E$ for all $e \in E\left(H^{\prime}\right)$, and so $w\left(v_{0}\right)+\sum_{v \in e} w(v) \geq 1$.

Hence the size of $w$ is at least an, so by Proposition 2.1 the largest fractional matching in $G$ has size at least an.

The proof of Theorem 1.8 proceeds as follows. Suppose $G$ has no fractional matching of size an. Then we use Lemma 3.1 and induction to show that $G$ contains few vertices of high degree. Moreover, by duality we show that $G$ has a small fractional vertex cover. We combine these two facts to show that the number of edges of $G$ does not exceed the expression stated in Theorem 1.8.

Proof of Theorem 1.8. The proof will proceed by induction on $k$. The base step, $k=2$, follows by Theorem 2.4 (as $0.48 / 2<1 / 3$ ).

Now consider some $k>2$ and suppose that the theorem holds for all smaller values of $k$. Let $\varepsilon>0$ and let $n_{0} \in \mathbb{N}$ be sufficiently large compared to $1 / \varepsilon$ and $k$. Fix any $a$ with $0<a \leq 0.48 / k$. Recall that by Proposition 2.6, $a_{k}^{\prime}>0.48 / k$, so then $a<a_{k}^{\prime}$. For convenience let us define

$$
\xi(a):=\left(1-\left(1-\frac{a}{1-a}\right)^{k-1}\right)(1-a)^{k-1}+1-(1-a)^{k-1}=1-(1-2 a)^{k-1} .
$$

Consider any $k$-uniform hypergraph $G=(V, E)$ on $n \geq n_{0}$ vertices, and suppose that the largest fractional matching of $G$ is of size less than an. Then by Proposition 2.1 there exists a fractional vertex cover, $w$ say, of $G$ with size less than $a n$. Note that

$$
\frac{a}{1-a} \leq \frac{0.48}{k(1-0.48 / k)}<\frac{0.48}{k-1}
$$

Let $c:=1-(1-a /(1-a))^{k-1}+\varepsilon / 2$. Then by induction and (3.2),

$$
f_{0}^{a n^{\prime} /(1-a)}\left(k-1, n^{\prime}\right) \leq c\left(\begin{array}{c}
n^{\prime} \\
k-1
\end{array}\right)
$$

for all sufficiently large $n^{\prime}$. So, as $n_{0}$ is sufficiently large, Lemma 3.1 implies that there are less than an vertices of $G$ with degree at least $(\xi(a)+\varepsilon)\left(\begin{array}{l}n-1 \\ k-1\end{array}\right)$.

Let $S$ be the set of $\lceil a n\rceil-1$ vertices of $G$ with highest degree. Note then that $d_{G}(v)<$ $(\xi(a)+\varepsilon)\left(\begin{array}{l}n-1 \\ k-1\end{array}\right)$ for all $v \in V \backslash S$. Given $X \subseteq V \backslash S$, for all $s \in S$ let $t_{X}(s)$ denote the number of 
$k$-tuples of vertices of $G$ consisting of $s$ and $k-1$ vertices from $V \backslash S$ such that at least one of these $k-1$ vertices lies in $X$.

We claim that $X \subseteq V \backslash S$ can be chosen such that $t_{X}(s) \geq \xi(a)\left(\begin{array}{l}n-1 \\ k-1\end{array}\right)$ for all $s \in S$. Indeed, if we take $X$ to be $V \backslash S$ then for each $s \in S$ we have that

$$
t_{X}(s)=\left(\begin{array}{c}
|X| \\
k-1
\end{array}\right)=\left((1-a)^{k-1}+o(1)\right)\left(\begin{array}{l}
n-1 \\
k-1
\end{array}\right) \geq \xi(a)\left(\begin{array}{l}
n-1 \\
k-1
\end{array}\right) .
$$

To see that the final inequality holds for sufficiently large $n_{0}$, note that

$$
\frac{\xi(a)}{(1-a)^{k-1}}=g_{k}(a)<g_{k}\left(a_{k}^{\prime}\right)=1
$$

by definition of $a$ and $a_{k}^{\prime}$, and the fact that $g_{k}(x)$ is strictly increasing for $x \in(0,1 /(k+1))$.

Choose $X \subseteq V \backslash S$ of minimal size with the property that $t_{X}(s) \geq \xi(a)\left(\begin{array}{l}n-1 \\ k-1\end{array}\right)$ for all $s \in S$. Note that $t_{X}(s)=t_{X}\left(s^{\prime}\right)$ for all $s, s^{\prime} \in S$ and $t_{X}(s) \leq(\xi(a)+\varepsilon)\left(\begin{array}{l}n-1 \\ k-1\end{array}\right)$. (The latter holds since we may assume that $n_{0}$ is sufficiently large.) Also let

$$
E^{\prime}:=\{e \in E: e \cap S=\emptyset\} \cup\{e \in E:|e \cap S|=1,|e \cap X| \geq 1\} .
$$

So

$$
\left|E \backslash E^{\prime}\right| \leq\left(\begin{array}{l}
n \\
k
\end{array}\right)-\left((1-a)^{k}+o(1)\right)\left(\begin{array}{l}
n \\
k
\end{array}\right)-\xi(a)\left(\begin{array}{l}
n-1 \\
k-1
\end{array}\right)(\lceil a n\rceil-1) .
$$

Now, note that by Proposition 2.2,

$$
e(G) \leq \sum_{e \in E} \sum_{v \in e \backslash S} w(v)+\sum_{e \in E^{\prime}} \sum_{v \in e \cap S} w(v)+\left|E \backslash E^{\prime}\right| .
$$

Together with the facts that $d_{G}(v)<(\xi(a)+\varepsilon)\left(\begin{array}{l}n-1 \\ k-1\end{array}\right)$ for all $v \in V \backslash S$ and that the number of edges in $E^{\prime}$ incident to $s$ is at most $t_{X}(s) \leq(\xi(a)+\varepsilon)\left(\begin{array}{l}n-1 \\ k-1\end{array}\right)$ for all $s \in S$, this implies that

$$
e(G) \leq \sum_{v \in V}(\xi(a)+\varepsilon)\left(\begin{array}{l}
n-1 \\
k-1
\end{array}\right) w(v)+\left|E \backslash E^{\prime}\right| .
$$

Now, recalling that the size of $w$ is less than an and that $a \leq 0.48 / k$ gives

$$
e(G)<(\xi(a)+\varepsilon)\left(\begin{array}{l}
n-1 \\
k-1
\end{array}\right) a n+\left|E \backslash E^{\prime}\right| \stackrel{(3.4)}{\leq}\left(1-(1-a)^{k}+\varepsilon\right)\left(\begin{array}{l}
n \\
k
\end{array}\right) .
$$

By definition, this shows that $f_{0}^{a n}(k, n) \leq\left(1-(1-a)^{k}+o(1)\right)\left(\begin{array}{l}n \\ k\end{array}\right)$. This, along with the lower bound (2.1), completes the inductive step and hence the proof.

Note that the main constraint which restricts the range of $a$ in our result is given by (3.3).

Proof of Theorem 1.10. The proof will proceed by induction on $k$. The base step, $k=2$, follows by Theorem 2.4, setting $x:=1 /(2+d)$.

Now consider some $k>2$ and suppose that the theorem holds for all smaller values of $k$. Fix $d \geq 1$. Let $\varepsilon>0$ and let $n_{0} \in \mathbb{N}$ be sufficiently large compared to $1 / \varepsilon, k$ and $d$. For convenience let us define

$$
\xi:=\left(\frac{k-1}{k+d-1}-\frac{k-2}{(k+d-1)^{k-1}}\right)\left(\frac{k+d-1}{k+d}\right)^{k-1}+\left(1-\left(\frac{k+d-1}{k+d}\right)^{k-1}\right)<1 .
$$


Consider any $k$-uniform hypergraph $G=(V, E)$ on $n \geq n_{0}$ vertices, and suppose that the largest fractional matching of $G$ is of size less than $n /(k+d)$. Then by Proposition 2.1 there exists a fractional vertex cover, $w$ say, of $G$ with size less than $n /(k+d)$. Let $a:=1 /(k+d)$. So $a /(1-a)=1 /(k+d-1)$. Let

$$
c:=\frac{k-1}{k+d-1}-\frac{k-2}{(k+d-1)^{k-1}}+\varepsilon / 4 .
$$

Then by induction,

$$
f_{0}^{n^{\prime} /(k+d-1)}(k-1, n) \leq c\left(\begin{array}{c}
n^{\prime} \\
k-1
\end{array}\right),
$$

for all sufficiently large $n^{\prime}$. Thus, as $n_{0}$ is sufficiently large, Lemma 3.1 implies that there are less than $n /(k+d)$ vertices of $G$ with degree at least $(\xi+\varepsilon / 2)\left(\begin{array}{l}n-1 \\ k-1\end{array}\right)$.

Let $S$ be the set of $|S|$ vertices of $G$ with highest degree, where $|S| \in k ! \mathbb{N}$ is minimal such that $|S| \geq n /(k+d)$. So $d_{G}(v)<(\xi+\varepsilon / 2)\left(\begin{array}{l}n-1 \\ k-1\end{array}\right)$ for all $v \in V \backslash S$. For every $i \in\{0, \ldots, k\}$ let $S_{i}:=\left\{e \in\left(\begin{array}{l}V \\ k\end{array}\right):|e \cap S|=i\right\}$. Given $X \subseteq\left(\begin{array}{l}V \\ k\end{array}\right)$, for all $v \in V$ let $t_{X}(v):=|\{e \in X: v \in e\}|$. Note that for all $v \in S$ the value of $t_{S_{i}}(v)$ is the same and $t_{S_{0}}(v)=0$. Let $\ell \in\{0, \ldots, k\}$ be maximal such that for any $v \in S$ we have $\sum_{i=0}^{\ell-1} t_{S_{i}}(v) \leq \xi\left(\begin{array}{l}n-1 \\ k-1\end{array}\right)$. Let $E^{\prime \prime \prime}:=\left(\begin{array}{l}V \\ k\end{array}\right) \backslash S_{k}$. Then for each $v \in S$,

$$
t_{E^{\prime \prime \prime}}(v)=\left(1-\frac{1}{(k+d)^{k-1}}+o(1)\right)\left(\begin{array}{l}
n-1 \\
k-1
\end{array}\right)>\xi\left(\begin{array}{l}
n-1 \\
k-1
\end{array}\right) .
$$

The final inequality holds here for sufficiently large $n_{0}$, as it rearranges to $d(k+d-1)^{k-2}+(k-$ $2)+o(1)>1$. This shows that $\ell \leq k-1$. Let

$$
\eta:=\left(\xi\left(\begin{array}{c}
n-1 \\
k-1
\end{array}\right)-\sum_{i=1}^{\ell-1} t_{S_{i}}(v)\right) /\left(\begin{array}{c}
|S| \\
\ell-1
\end{array}\right)\left(\begin{array}{c}
n-|S| \\
k-\ell
\end{array}\right) .
$$

So $\eta \in[0,1)$. Apply Proposition 2.8 with parameters $n, k, \ell, \eta$ to obtain a set $\tilde{E} \subseteq S_{\ell}$ such that for every $v \in S$,

$$
t_{\tilde{E}}(v)=\left\lfloor\eta\left(\begin{array}{c}
|S| \\
\ell-1
\end{array}\right)\left(\begin{array}{c}
n-|S| \\
k-\ell
\end{array}\right)\right\rfloor .
$$

Let $E^{\prime \prime}:=\bigcup_{i=0}^{\ell-1} S_{i} \cup \tilde{E}$. Then each $v \in S$ satisfies

$$
t_{E^{\prime \prime}}(v)=\left\lfloor\xi\left(\begin{array}{l}
n-1 \\
k-1
\end{array}\right)\right\rfloor .
$$

We can now give a lower bound on the size of $E^{\prime \prime}$ as follows: for each vertex $v \in S$ we count the number of $k$-tuples in $E^{\prime \prime}$ that contain $v$, and then adjust for the $k$-tuples that contain several vertices of $S$ and were thus counted several times as a result. Since $S_{0} \subseteq E^{\prime \prime}$ this yields

$$
\left|E^{\prime \prime}\right| \stackrel{(3.6)}{\geq}\left\lfloor\xi\left(\begin{array}{l}
n-1 \\
k-1
\end{array}\right)\right\rfloor \frac{n}{k+d}+\left|S_{0}\right|-\sum_{j=1}^{k-1}(j-1)\left|S_{j}\right| .
$$


Note that since $E^{\prime \prime} \subseteq E^{\prime \prime \prime}$ we only need to consider values of $j$ up to $k-1$ in the summation, rather than $k$. Now, note that

$$
\begin{aligned}
\left|S_{0}\right|-\sum_{j=1}^{k}(j-1)\left|S_{j}\right| & =\left(\begin{array}{l}
n \\
k
\end{array}\right)-\sum_{v \in S}\left(\begin{array}{l}
n-1 \\
k-1
\end{array}\right)=\left(\begin{array}{l}
n \\
k
\end{array}\right)-\left(\frac{n}{k+d}+o(1)\right)\left(\begin{array}{l}
n-1 \\
k-1
\end{array}\right) \\
& =\left(1-\frac{k}{k+d}+o(1)\right)\left(\begin{array}{l}
n \\
k
\end{array}\right) .
\end{aligned}
$$

Hence, as $(k-1)\left|S_{k}\right|=\left((k-1) /(k+d)^{k}+o(1)\right)\left(\begin{array}{l}n \\ k\end{array}\right)$,

$$
\left|E^{\prime \prime}\right| \geq(\xi+o(1))\left(\begin{array}{l}
n-1 \\
k-1
\end{array}\right) \frac{n}{k+d}+\left(1-\frac{k}{k+d}+\frac{k-1}{(k+d)^{k}}+o(1)\right)\left(\begin{array}{l}
n \\
k
\end{array}\right) .
$$

Now, let $E^{\prime}:=E \cap E^{\prime \prime}$. Also, note that by Proposition 2.2,

$$
e(G) \leq \sum_{e \in E} \sum_{v \in e \backslash S} w(v)+\sum_{e \in E^{\prime}} \sum_{v \in e \cap S} w(v)+\left|E \backslash E^{\prime}\right| .
$$

Recall that $d_{G}(v)<(\xi+\varepsilon / 2)\left(\begin{array}{c}n-1 \\ k-1\end{array}\right)$ for all $v \in V \backslash S$ and that by (3.6) the number of edges in $E^{\prime}$ incident to $v$ is at most $\xi\left(\begin{array}{l}n-1 \\ k-1\end{array}\right)$ for all $v \in S$. So

$$
e(G) \leq \sum_{v \in V}(\xi+\varepsilon / 2)\left(\begin{array}{l}
n-1 \\
k-1
\end{array}\right) w(v)+\left|E \backslash E^{\prime}\right| .
$$

Now note that $\left|E \backslash E^{\prime}\right| \leq\left|\left(\begin{array}{l}V \\ k\end{array}\right) \backslash E^{\prime \prime}\right|=\left(\begin{array}{l}n \\ k\end{array}\right)-\left|E^{\prime \prime}\right|$ and recall that the size of $w$ is less than $n /(k+d)$. So

$$
\begin{aligned}
e(G)< & (\xi+\varepsilon / 2)\left(\begin{array}{l}
n-1 \\
k-1
\end{array}\right) \frac{n}{k+d}+\left(\begin{array}{l}
n \\
k
\end{array}\right)-\left|E^{\prime \prime}\right| \\
\stackrel{(3.7)}{\leq} & (\xi+\varepsilon / 2)\left(\begin{array}{l}
n-1 \\
k-1
\end{array}\right) \frac{n}{k+d}-(\xi+o(1))\left(\begin{array}{l}
n-1 \\
k-1
\end{array}\right) \frac{n}{k+d} \\
& +\left(\frac{k}{k+d}-\frac{k-1}{(k+d)^{k}}+o(1)\right)\left(\begin{array}{l}
n \\
k
\end{array}\right) \\
\leq & \left(\frac{k}{k+d}-\frac{k-1}{(k+d)^{k}}+\varepsilon\right)\left(\begin{array}{l}
n \\
k
\end{array}\right) .
\end{aligned}
$$

The final inequality holds since $n_{0}$ is sufficiently large. By definition, this shows that

$$
f_{0}^{n /(k+d)}(k, n) \leq\left(\frac{k}{k+d}-\frac{k-1}{(k+d)^{k}}+o(1)\right)\left(\begin{array}{l}
n \\
k
\end{array}\right) .
$$

This completes the inductive step and hence the proof.

\section{Minimum Degree CONDitions FOR FraCtional Matchings}

The following proposition generalises Proposition 1.1 in [1], with a similar proof idea. It allows us to transform bounds involving edge densities into bounds involving $d$-degrees. 
Proposition 4.1. Let $\varepsilon \geq 0$, let $k, d$, $n$ be integers with $n \geq k \geq 3,1 \leq d \leq k-2$, and $d<\left(1-\varepsilon^{1 / d}\right) n$. Let $a \in\left[0,\left(1-\varepsilon^{1 / d}\right) / k\right]$. Suppose $H$ is a $k$-uniform hypergraph on $n$ vertices, such that for at least $(1-\varepsilon)\left(\begin{array}{l}n \\ d\end{array}\right)$ d-tuples of vertices $L \in\left(\begin{array}{c}V(H) \\ d\end{array}\right)$ we have

$$
\operatorname{deg}_{H}(L) \geq f_{0}^{a n}(k-d, n-d) .
$$

Then $H$ has a fractional matching of size an.

Proof. The outline of the proof goes as follows. We will assume that there is no fractional matching of size an in a $k$-uniform hypergraph $H=(V, E)$ on $n$ vertices and then show that for more than $\varepsilon\left(\begin{array}{l}n \\ d\end{array}\right) d$-tuples of vertices $L \in\left(\begin{array}{l}V \\ d\end{array}\right)$, the neighbourhood hypergraph $H(L)$ of $L$ in $H$ has no fractional matching of size an. This will imply that for more than $\varepsilon\left(\begin{array}{l}n \\ d\end{array}\right) d$-tuples of vertices $L, \operatorname{deg}_{H}(L)=e(H(L))<f_{0}^{a n}(k-d, n-d)$. This will prove the result in contrapositive.

So suppose $H=(V, E)$ is an $n$-vertex $k$-uniform hypergraph, with no fractional matching of size an. Then by Proposition 2.1, $H$ has a fractional vertex cover, $w$ say, of size less than an. Let

$$
E_{w}:=\left\{e \in\left(\begin{array}{l}
V \\
k
\end{array}\right): \sum_{v \in e} w(v) \geq 1\right\}
$$

and let $H_{w}:=\left(V, E_{w}\right)$. Since $H \subseteq H_{w}$ we can, without loss of generality, replace $H$ with $H_{w}$. Let $U \subseteq V$ be the set of $\left\lfloor\varepsilon^{1 / d} n\right\rfloor+d$ vertices of smallest weights. Let $\mathcal{L}:=\left(\begin{array}{c}U \\ d\end{array}\right)$. Note that

$$
|\mathcal{L}|=\left(\begin{array}{c}
\left\lfloor\varepsilon^{1 / d} n\right\rfloor+d \\
d
\end{array}\right)>\frac{\left(\varepsilon^{1 / d} n\right)^{d}}{d !}=\varepsilon \frac{n^{d}}{d !} \geq \varepsilon\left(\begin{array}{c}
n \\
d
\end{array}\right) .
$$

Consider any $L \in \mathcal{L}$. Let $H_{w}(L)$ be the neighbourhood hypergraph of $L$ in $H_{w}$. We will show that $H_{w}(L)$ has no fractional matching of size an. Without loss of generality we may assume that the elements of $L$ all have equal weights, $w(L)$ say. (If not, we could replace these weights by their average, which would alter neither $\sum_{v \in V} w(v)$ nor $\sum_{v \in e} w(v)$ for any $e \supseteq L$. These are the only two quantities involving weights that we will consider in what follows.) Observe that $w(L)<1 / k$, else the size of $w$ would be at least

$$
\frac{n\left(1-\varepsilon^{1 / d}\right)}{k} \geq a n
$$

We now define a new weight function $w^{\prime}(v)$ on the vertices in $V$ :

$$
w^{\prime}(v):=\min \left\{\max \left\{0, w^{*}(v)\right\}, 1\right\}, \quad \text { where } w^{*}(v):=\frac{w(v)-w(L)}{1-k w(L)} .
$$

Note that only for vertices $u \in U \backslash L$ can it be that $w^{*}(u)<0$. Note also that since $w(v) \geq 0$ for all $v \in V$, we have that $w^{*}(u) \geq-w(L) /(1-k w(L))$ for such vertices $u$. Hence,

$$
\begin{aligned}
\sum_{v \in V} w^{\prime}(v) & \leq\left(\sum_{v \in V} w^{*}(v)\right)+|U \backslash L| \frac{w(L)}{1-k w(L)}<\frac{a n-n w(L)+\varepsilon^{1 / d} n w(L)}{1-k w(L)} \\
& =a n \frac{1-(1 / a)\left(1-\varepsilon^{1 / d}\right) w(L)}{1-k w(L)} \leq a n,
\end{aligned}
$$

and for any given $e \in\left\{e^{\prime} \in E_{w}: e^{\prime} \supseteq L\right\}$ we have that

$$
\sum_{v \in e} w^{\prime}(v) \geq \min \left\{\frac{\sum_{v \in e} w(v)-k w(L)}{1-k w(L)}, 1\right\} \geq \min \left\{\frac{1-k w(L)}{1-k w(L)}, 1\right\}=1 .
$$


Moreover, $\sum_{v \in L} w^{\prime}(v)=0$. It follows that the function $w^{\prime}$ restricted to $V \backslash L$ is a fractional vertex cover of $H_{w}(L)$ of size less than an, and so by Proposition 2.1, $H_{w}(L)$ has no fractional matching of size an, which completes the proof.

We can now derive Theorems 1.9 and 1.11.

Proof of Theorem 1.9. Let $k^{\prime}:=k-d$ and $n^{\prime}:=n-d$. Then Theorem 1.8 and Proposition 2.3 together imply that

$$
\begin{aligned}
f_{0}^{a n}(k-d, n-d) & =f_{0}^{a\left(n^{\prime}+d\right)}\left(k^{\prime}, n^{\prime}\right) \leq f_{0}^{a n^{\prime}+1}\left(k^{\prime}, n^{\prime}\right) \leq\left(1-(1-a)^{k^{\prime}}+o(1)\right)\left(\begin{array}{l}
n^{\prime} \\
k^{\prime}
\end{array}\right) \\
& =\left(1-(1-a)^{k-d}+o(1)\right)\left(\begin{array}{l}
n-d \\
k-d
\end{array}\right) .
\end{aligned}
$$

The upper bound in Theorem 1.9 follows now from Proposition 4.1 applied with $\varepsilon=0$. The lower bound follows from (2.1).

Proof of Theorem 1.11. Let $k^{\prime}:=k-d$ and $n^{\prime}:=n-d$. Then Theorem 1.10 and Proposition 2.3 together imply that

$$
\begin{aligned}
f_{0}^{n / k}(k-d, n-d) & =f_{0}^{\left(n^{\prime}+d\right) /\left(k^{\prime}+d\right)}\left(k^{\prime}, n^{\prime}\right) \leq f_{0}^{n^{\prime} /\left(k^{\prime}+d\right)+1}\left(k^{\prime}, n^{\prime}\right) \\
& \leq\left(\frac{k^{\prime}}{k^{\prime}+d}-\frac{k^{\prime}-1}{\left(k^{\prime}+d\right)^{k^{\prime}}}+o(1)\right)\left(\begin{array}{l}
n^{\prime} \\
k^{\prime}
\end{array}\right) \\
& =\left(\frac{k-d}{k}-\frac{k-d-1}{k^{k-d}}+o(1)\right)\left(\begin{array}{l}
n-d \\
k-d
\end{array}\right) .
\end{aligned}
$$

So Theorem 1.11 follows now from Proposition 4.1 applied with $\varepsilon=0$.

The case $\varepsilon>0$ of Proposition 4.1 will be used in the next section.

\section{Constructing integer matchings From fractional Ones}

We will construct integer matchings from fractional ones using the Weak Hypergraph Regularity Lemma. Before stating this we will need the following definitions.

Given a $k$-tuple $\left(V_{1}, \ldots, V_{k}\right)$ of disjoint subsets of the vertices of a $k$-uniform hypergraph $G=(V, E)$, we define $\left(V_{1}, \ldots, V_{k}\right)_{G}$ to be the $k$-partite subhypergraph with vertex classes $V_{1}, \ldots, V_{k}$ induced on $G$. We let

$$
d_{G}\left(V_{1}, \ldots, V_{k}\right)=\frac{e\left(\left(V_{1}, \ldots, V_{k}\right)_{G}\right)}{\prod_{i \in\{1, \ldots, k\}}\left|V_{i}\right|}
$$

denote the density of $\left(V_{1}, \ldots, V_{k}\right)_{G}$.

Definition 5.1 ( $\varepsilon$-regularity). Let $\varepsilon>0$, let $G=(V, E)$ be a $k$-uniform hypergraph, and let $V_{1}, \ldots, V_{k} \subseteq V$ be disjoint. We say that $\left(V_{1}, \ldots, V_{k}\right)_{G}$ is $\varepsilon$-regular if for every subhypergraph $\left(V_{1}^{\prime}, \ldots, V_{k}^{\prime}\right)_{G}$ with $V_{i}^{\prime} \subseteq V_{i}$ and $\left|V_{i}^{\prime}\right| \geq \varepsilon\left|V_{i}\right|$ for each $i \in\{1, \ldots, k\}$, we have that

$$
\left|d_{G}\left(V_{1}^{\prime}, \ldots, V_{k}^{\prime}\right)-d_{G}\left(V_{1}, \ldots, V_{k}\right)\right|<\varepsilon
$$

The following result was proved by Chung [5]. The proof follows the lines of that of the original Regularity Lemma for graphs [30]. 
Lemma 5.2 (Weak Hypergraph Regularity Lemma). For all integers $k \geq 2, L_{0} \geq 1$, and every $\varepsilon>0$ there exists $N=N\left(\varepsilon, L_{0}, k\right)$ such that if $G=(V, E)$ is a $k$-uniform hypergraph on $n \geq N$ vertices, then $V$ has a partition $V_{0}, \ldots, V_{L}$ such that the following properties hold:

(i) $L_{0} \leq L \leq N$ and $\left|V_{0}\right| \leq \varepsilon n$,

(ii) $\left|V_{1}\right|=\cdots=\left|V_{L}\right|$,

(iii) for all but at most $\varepsilon\left(\begin{array}{c}L \\ k\end{array}\right) k$-tuples $\left\{i_{1}, \ldots, i_{k}\right\} \in\left(\begin{array}{c}{[L]} \\ k\end{array}\right)$, we have that $\left(V_{i_{1}}, \ldots, V_{i_{k}}\right)_{G}$ is $\varepsilon$ regular.

We call the partition classes $V_{1}, \ldots, V_{L}$ clusters, and $V_{0}$ the exceptional set. For our purposes we will in fact use the degree form of the Weak Hypergraph Regularity Lemma.

Lemma 5.3 (Degree Form of the Weak Hypergraph Regularity Lemma). For all integers $k \geq 2$, $L_{0} \geq 1$ and every $\varepsilon>0$, there is an $N=N\left(\varepsilon, L_{0}, k\right)$ such that for every $d \in[0,1)$ and for every hypergraph $G=(V, E)$ on $n \geq N$ vertices there exists a partition of $V$ into $V_{0}, V_{1}, \ldots, V_{L}$ and a spanning subhypergraph $G^{\prime}$ of $G$ such that the following properties hold:

(i) $L_{0} \leq L \leq N$ and $\left|V_{0}\right| \leq \varepsilon n$,

(ii) $\left|V_{1}\right|=\cdots=\left|V_{L}\right|=: m$,

(iii) $d_{G^{\prime}}(v)>d_{G}(v)-(d+\varepsilon) n^{k-1}$ for all $v \in V$,

(iv) every edge of $G^{\prime}$ with more than one vertex in a single cluster $V_{i}$, for some $i \in\{1, \ldots, L\}$, has at least one vertex in $V_{0}$,

(v) for all $k$-tuples $\left\{i_{1}, \ldots, i_{k}\right\} \in\left(\begin{array}{c}{[L]} \\ k\end{array}\right)$, we have that $\left(V_{i_{1}}, \ldots, V_{i_{k}}\right)_{G^{\prime}}$ is $\varepsilon$-regular and has density either 0 or $>d$.

The proof is very similar to that of the degree form of the Regularity Lemma for graphs, so we omit it here; for details see [31].

We now define a type of hypergraph that will be essential in our application of the Weak Hypergraph Regularity Lemma.

Definition 5.4 (Reduced Hypergraph). Let $G=(V, E)$ be a k-uniform hypergraph. Given parameters $\varepsilon>0, d \in[0,1)$ and $L_{0} \geq 1$ we define the reduced hypergraph $R=R\left(\varepsilon, d, L_{0}\right)$ of $G$ as follows. Apply the degree form of the Weak Hypergraph Regularity Lemma to $G$, with parameters $\varepsilon, d, L_{0}$ to obtain a spanning subhypergraph $G^{\prime}$ and a partition $V_{0}, \ldots, V_{L}$ of $V$, with exceptional set $V_{0}$ and clusters $V_{1}, \ldots, V_{L}$. Then $R$ has vertices $V_{1}, \ldots, V_{L}$, and there exists an edge between $V_{i_{1}}, \ldots, V_{i_{k}}$ precisely when $\left(V_{i_{1}}, \ldots, V_{i_{k}}\right)_{G^{\prime}}$ is $\varepsilon$-regular with density greater than $d$.

The following lemma tells us that this reduced hypergraph (almost) inherits the edge density and minimum degree properties of the original hypergraph. The proof is similar to that of the well known versions for graphs, but we include it here for completeness.

\section{Lemma 5.5.}

(i) Suppose $c>0, k \geq 2, L_{0} \geq 1$ and $0<\varepsilon \leq d \leq c / 4$. Let $G$ be a k-uniform hypergraph with $e(G) \geq c|G|^{k}$. Let $R=R\left(\varepsilon, d, L_{0}\right)$ be the reduced hypergraph of $G$. Then $e(R) \geq$ $(c-4 d)|R|^{k}$.

(ii) Suppose $c>0, k \geq 2,1 \leq \ell \leq k-1, L_{0} \geq 1$, and $0<\varepsilon \leq d \leq c^{3} / 64$. Let $G$ be a $k$-uniform hypergraph with $\delta_{\ell}(G) \geq c|G|^{k-\ell}$. Let $R=R\left(\varepsilon, d, L_{0}\right)$ be the reduced hypergraph of $G$. Then at least $\left(\begin{array}{c}|R| \\ \ell\end{array}\right)-d^{1 / 3}(2 k)^{\ell}|R|^{\ell}$ of the $\ell$-tuples of vertices of $R$ have degree at least $\left(c-4 d^{1 / 3}\right)|R|^{k-\ell}$. 
Proof. Throughout this proof let $G^{\prime}$ be the spanning subhypergraph of $G$ obtained by applying the degree form of the Weak Hypergraph Regularity Lemma to $G$ with parameters $\varepsilon, d, L_{0}$; let $V_{1}, \ldots, V_{L}$ denote the vertices of $R$, and let $m$ denote the size of these clusters.

To prove (i), suppose that $e(R)<(c-4 d)|R|^{k}$. So there are less than $(c-4 d)|R|^{k} k$-tuples $\left\{i_{1}, \ldots, i_{k}\right\} \in\left(\begin{array}{c}{[L]} \\ k\end{array}\right)$ such that $\left(V_{i_{1}}, \ldots, V_{i_{k}}\right)_{G^{\prime}}$ has non-zero density. Note that at most $m^{k}$ edges lie in such a $k$-tuple. So

$$
e\left(G^{\prime}-V_{0}\right)<(c-4 d)|R|^{k} m^{k} \leq(c-4 d)|G|^{k} .
$$

However, given any vertex $x \in V\left(G^{\prime}\right)$ we know that $d_{G^{\prime}}(x)>d_{G}(x)-(d+\varepsilon)|G|^{k-1}$. Note that since $\left|V_{0}\right| \leq \varepsilon|G|$, we have that the number of edges incident to $x$ that contain a vertex in $V_{0}$ is at most $\varepsilon|G|\left(\begin{array}{c}|G| \\ k-2\end{array}\right) \leq \varepsilon|G|^{k-1}$. Hence for all $v \in V\left(G^{\prime}-V_{0}\right)$, we have that $d_{G^{\prime}-V_{0}}(v)>$ $d_{G}(v)-(d+2 \varepsilon)|G|^{k-1}$. So

$$
e\left(G^{\prime}-V_{0}\right)=\frac{\sum_{v \in V\left(G^{\prime}-V_{0}\right)} d_{G^{\prime}-V_{0}}(v)}{k}>\frac{\left(\sum_{v \in V(G)} d_{G}(v)\right)-\left(\sum_{v \in V_{0}} d_{G}(v)\right)-(d+2 \varepsilon)|G|^{k}}{k} .
$$

Since $\left|V_{0}\right| \leq \varepsilon|G|$, this implies that

$$
e\left(G^{\prime}-V_{0}\right)>e(G)-\frac{(d+3 \varepsilon)|G|^{k}}{k}>(c-4 d)|G|^{k},
$$

a contradiction. This proves (i).

To prove (ii), recall from the proof of (i) that for any vertex $v \in V\left(G^{\prime}-V_{0}\right)$ we have that

$$
d_{G^{\prime}-V_{0}}(v)>d_{G}(v)-(d+2 \varepsilon)|G|^{k-1} \geq d_{G}(v)-3 d|G|^{k-1} .
$$

We call an $\ell$-tuple $A$ of vertices of $G^{\prime}-V_{0}$ bad if $\operatorname{deg}_{G^{\prime}-V_{0}}(A) \leq \operatorname{deg}_{G}(A)-3 d^{1 / 3}|G|^{k-\ell}$. So for each $v \in V\left(G^{\prime}-V_{0}\right)$ there are at most $\left(\begin{array}{c}k-1 \\ \ell-1\end{array}\right) d^{2 / 3}|G|^{\ell-1}$ bad $\ell$-tuples $A$ with $v \in A$. (This follows by double-counting the number of pairs $(A, e)$ where $A$ is a bad $\ell$-tuple with $v \in A$ and $e \in E(G) \backslash E\left(G^{\prime}-V_{0}\right)$ is an edge containing $A$.) This in turn implies that in total at most $\left(\begin{array}{c}k-1 \\ \ell-1\end{array}\right) d^{2 / 3}|G|^{\ell}$ of the $\ell$-tuples $A$ are bad. Given $1 \leq s \leq k$ and an $s$-tuple $\left(V_{i_{1}}, \ldots, V_{i_{s}}\right)$ of clusters of $R$, we say that an $s$-tuple $A$ of vertices of $G^{\prime}-V_{0}$ lies in $\left(V_{i_{1}}, \ldots, V_{i_{s}}\right)$ if $\left|A \cap V_{i_{\alpha}}\right|=1$ for all $\alpha \in\{1, \ldots, s\}$. We call an $\ell$-tuple $\left(V_{i_{1}}, \ldots, V_{i_{\ell}}\right)$ of clusters of $R$ nice if there are less than $d^{1 / 3} m^{\ell}$ bad $\ell$-tuples $A$ of vertices of $G^{\prime}-V_{0}$ which lie in $\left(V_{i_{1}}, \ldots, V_{i_{\ell}}\right)$. So less than $\left(\begin{array}{c}k-1 \\ \ell-1\end{array}\right) d^{1 / 3}|G|^{\ell} / m^{\ell} \leq d^{1 / 3}(2 k)^{\ell}|R|^{\ell}$ of the $\ell$-tuples of clusters of $R$ are not nice. Hence it suffices to show that any nice $\ell$-tuple of clusters of $R$ has degree at least $\left(c-4 d^{1 / 3}\right)|R|^{k-\ell}$ in $R$.

Consider any nice $\ell$-tuple of clusters of $R$, say $\left(V_{i_{1}}, \ldots, V_{i_{\ell}}\right)$. Let $\mathcal{A}$ denote the set of all $\ell$ tuples $A$ of vertices of $G^{\prime}-V_{0}$ which lie in $\left(V_{i_{1}}, \ldots, V_{i_{\ell}}\right)$ and are not bad. So $|\mathcal{A}| \geq\left(1-d^{1 / 3}\right) m^{\ell}$. Moreover, the number of edges $e$ of $G^{\prime}-V_{0}$ with $\left|e \cap V_{i_{\alpha}}\right|=1$ for all $\alpha \in\{1, \ldots, \ell\}$ is at least

$$
\sum_{A \in \mathcal{A}} \operatorname{deg}_{G^{\prime}-V_{0}}(A) \geq|\mathcal{A}|\left(c-3 d^{1 / 3}\right)|G|^{k-\ell} \geq\left(c-4 d^{1 / 3}\right)|G|^{k-\ell} m^{\ell} .
$$

Now suppose that the degree in $R$ of $\left(V_{i_{1}}, \ldots, V_{i_{\ell}}\right)$ is less than $\left(c-4 d^{1 / 3}\right)|R|^{k-\ell}$. Then the number of $(k-\ell)$-tuples $\left\{j_{1}, \ldots, j_{k-\ell}\right\} \in\left(\begin{array}{c}{[L]} \\ k-\ell\end{array}\right)$ for which $\left(V_{i_{1}}, \ldots, V_{i_{\ell}}, V_{j_{1}}, \ldots, V_{j_{k-\ell}}\right)_{G^{\prime}}$ is $\varepsilon$-regular with density greater than $d$ is less than $\left(c-4 d^{1 / 3}\right)|R|^{k-\ell}$. Note that at most $m^{k}$ edges of $G^{\prime}-V_{0}$ lie in such a subhypergraph. So the number of edges $e$ of $G^{\prime}-V_{0}$ with $\left|e \cap V_{i_{\alpha}}\right|=1$ for all $\alpha \in\{1, \ldots, \ell\}$ is less than

$$
\left(c-4 d^{1 / 3}\right)|R|^{k-\ell} m^{k} \leq\left(c-4 d^{1 / 3}\right)|G|^{k-\ell} m^{\ell},
$$


contradicting (5.1). This completes the proof.

The following lemma uses all of the previous results of this section to allow us to convert our fractional matchings into integer ones. We will use the notation $a \ll b$ to mean that we can find an increasing function $f$ for which all of the conditions in the proof are satisfied whenever $a \leq f(b)$.

Lemma 5.6. Let $k \geq 2$ and $1 \leq \ell \leq k-1$ be integers, and let $\varepsilon>0$.

(i) Suppose that for some $b, c \in(0,1)$ and some integer $n_{0}$, any $k$-uniform hypergraph $G^{*}$ on $n \geq n_{0}$ vertices with $e\left(G^{*}\right) \geq c n^{k}$ has a fractional matching of size $(b+\varepsilon) n$. Then there exists an integer $n_{0}^{\prime}$ such that any $k$-uniform hypergraph $G$ on $n \geq n_{0}^{\prime}$ vertices with $e(G) \geq(c+\varepsilon) n^{k}$ has an (integer) matching of size at least bn.

(ii) Suppose that for some $b, c \in(0,1)$ and some integer $n_{0}$, any $k$-uniform hypergraph on $n \geq n_{0}$ vertices with at least $(1-\varepsilon)\left(\begin{array}{l}n \\ \ell\end{array}\right)$-tuples of vertices of degree at least $\mathrm{cn}^{k-\ell}$ has a fractional matching of size $(b+\varepsilon) n$. Then there exists an integer $n_{0}^{\prime}$ such that any $k$-uniform hypergraph $G$ on $n \geq n_{0}^{\prime}$ vertices with $\delta_{\ell}(G) \geq(c+\varepsilon) n^{k-\ell}$ has an (integer) matching of size at least bn.

Proof. We only prove (ii) here; the proof of (i) is very similar (using Lemma 5.5(i) rather than (ii)). Define $n_{0}^{\prime} \in \mathbb{N}$ and new constants $\varepsilon^{\prime}$ and $d$ such that $0<1 / n_{0}^{\prime} \ll \varepsilon^{\prime} \ll d \ll \varepsilon, c, 1 / k, 1 / n_{0}$. Let $G$ be a $k$-uniform hypergraph on $n \geq n_{0}^{\prime}$ vertices, with $\delta_{\ell}(G) \geq(c+\varepsilon) n^{k-1}$. Let $G^{\prime}$ be the spanning subhypergraph of $G$ obtained by applying the degree form of the Weak Hypergraph Regularity Lemma to $G$ with parameters $\varepsilon^{\prime}, d, n_{0}$. Let $R:=R\left(\varepsilon^{\prime}, d, n_{0}\right)$ be the corresponding reduced hypergraph, and let $L:=|R|$. By Lemma 5.5(ii) at least $(1-\varepsilon)\left(\begin{array}{c}L \\ \ell\end{array}\right) \ell$-tuples of vertices of $R$ have degree at least

$$
\left(c+\varepsilon-4 d^{1 / 3}\right) L^{k-\ell} \geq c L^{k-\ell} .
$$

So by the assumption in the statement of the lemma, $R$ has a fractional matching, $F$ say, of size $(b+\varepsilon) L$.

For each $e \in E(R)$, let $K_{e}:=\left\lceil\left(1-2 \varepsilon^{\prime}\right) F(e) m\right\rceil$, where $m$ is the size of each of the clusters of $R$. Now construct an integer matching, $M$ say, in $G$ by greedily adding to $M$ edges of $G^{\prime}$ until, for each $e=\left\{V_{j_{1}}, \ldots, V_{j_{k}}\right\} \in E(R), M$ contains precisely $K_{e}$ edges of $\left(V_{j_{1}}, \ldots, V_{j_{k}}\right)_{G^{\prime}}$. Note that at each stage of this process the number of vertices in each $V_{i} \in V(R)$ that would be covered by $M$ is at most

$$
\sum_{e: V_{i} \in e} K_{e} \leq \sum_{e: V_{i} \in e}\left(\left(1-2 \varepsilon^{\prime}\right) F(e) m+1\right) \leq\left(1-2 \varepsilon^{\prime}\right) m+\left(\begin{array}{l}
L-1 \\
k-1
\end{array}\right) \leq\left(1-\varepsilon^{\prime}\right) m .
$$

Note also that for every edge $e=\left\{V_{j_{1}}, \ldots, V_{j_{k}}\right\} \in E(R)$, we have that $\left(V_{j_{1}}, \ldots, V_{j_{k}}\right)_{G^{\prime}}$ is $\varepsilon^{\prime}$ regular with density $d>\varepsilon^{\prime}$. So indeed, by the definition of $\varepsilon^{\prime}$-regularity, it is possible to successively add edges to $M$ in order to obtain a matching $M$ as desired.

Note that the size of $M$ is

$$
\sum_{e \in E(R)} K_{e} \geq \sum_{e \in E(R)}\left(1-2 \varepsilon^{\prime}\right) F(e) m=\left(1-2 \varepsilon^{\prime}\right) m(b+\varepsilon) L \geq\left(1-2 \varepsilon^{\prime}\right)(b+\varepsilon)\left(1-\varepsilon^{\prime}\right) n \geq b n .
$$

So indeed $G$ has an (integer) matching of size at least $b n$. 
Now, using Lemma 5.6(i), Theorem 1.8 and the lower bound (2.1) immediately yields Theorem 1.2. We can also now prove Theorem 1.5.

Proof of Theorem 1.5. Let $\varepsilon^{\prime}>0$ and let $0<\varepsilon^{\prime \prime} \ll \varepsilon^{\prime}, \varepsilon, 1 / k, 0.48 /(k-d)-a$. Let $n_{0} \in \mathbb{N}$ be sufficiently large and suppose that $n \geq n_{0}$. Let $k^{\prime}:=k-d$ and $n^{\prime}:=n-d$. Then Theorem 1.8 implies that

$$
\begin{aligned}
f_{0}^{\left(a+\varepsilon^{\prime \prime}\right) n}(k-d, n-d) & =f_{0}^{\left(a+\varepsilon^{\prime \prime}\right)\left(n^{\prime}+d\right)}\left(k^{\prime}, n^{\prime}\right) \leq f_{0}^{\left(a+2 \varepsilon^{\prime \prime}\right) n^{\prime}}\left(k^{\prime}, n^{\prime}\right) \\
& \leq\left(1-\left(1-a-2 \varepsilon^{\prime \prime}\right)^{k^{\prime}}+\frac{\varepsilon^{\prime}}{4}\right)\left(\begin{array}{l}
n^{\prime} \\
k^{\prime}
\end{array}\right) \leq\left(1-(1-a)^{k-d}+\frac{\varepsilon^{\prime}}{2}\right)\left(\begin{array}{l}
n-d \\
k-d
\end{array}\right) .
\end{aligned}
$$

So by Proposition 4.1, if $H$ is a $k$-uniform hypergraph on $n$ vertices such that for at least $\left(1-\varepsilon^{\prime \prime}\right)\left(\begin{array}{l}n \\ d\end{array}\right) d$-tuples of vertices $L \in\left(\begin{array}{c}V(H) \\ d\end{array}\right)$ we have

$$
\operatorname{deg}_{H}(L) \geq \frac{1-(1-a)^{k-d}+\varepsilon^{\prime} / 2}{(k-d) !} n^{k-d} \geq\left(1-(1-a)^{k-d}+\frac{\varepsilon^{\prime}}{2}\right)\left(\begin{array}{l}
n-d \\
k-d
\end{array}\right),
$$

then $H$ has a fractional matching of size $\left(a+\varepsilon^{\prime \prime}\right) n$. So by Lemma 5.6(ii), any $k$-uniform hypergraph $G$ on $n \geq n_{0}^{\prime}$ vertices (where $n_{0}^{\prime}$ is sufficiently large) with

$$
\delta_{d}(G) \geq\left(1-(1-a)^{k-d}+\varepsilon^{\prime}\right)\left(\begin{array}{l}
n-d \\
k-d
\end{array}\right) \geq\left(\frac{1-(1-a)^{k-d}+\varepsilon^{\prime} / 2}{(k-d) !}+\varepsilon^{\prime \prime}\right) n^{k-d}
$$

has an (integer) matching of size at least an. This gives the upper bound in Theorem 1.5. The lower bound follows from (2.1).

We can prove Theorem 1.6 in a similar way, but to do so we will need to use the absorbing technique as introduced by Rödl, Ruciński and Szemerédi [29]. More precisely, we use the existence of a small and powerful matching $M_{a b s}$ in $G$ which, by 'absorbing' vertices, can transform any almost perfect matching into a perfect matching. $M_{a b s}$ has the property that whenever $X$ is a sufficiently small set of vertices of $G$ not covered by $M_{a b s}$ (and $|X| \in k \mathbb{N}$ ) there exists a matching in $G$ which covers precisely the vertices in $X \cup V\left(M_{a b s}\right)$. Since this part of the proof of Theorem 1.6 is very similar to the corresponding part of the proof of Theorem 1.1 in [1], we only sketch it.

Proof of Theorem 1.6 (sketch). Let $\varepsilon>0$ and suppose that $G$ is a $k$-uniform hypergraph on $n$ vertices with minimum $d$-degree at least

$$
\left(\frac{k-d}{k}-\frac{k-d-1}{k^{k-1}}+\varepsilon\right)\left(\begin{array}{l}
n-d \\
k-d
\end{array}\right)>\left(\frac{1}{2}+\varepsilon\right)\left(\begin{array}{l}
n-d \\
k-d
\end{array}\right)
$$

(5.2) implies that we can use the Strong Absorbing Lemma from [13] to find an absorbing matching $M_{a b s}$ in $G$, and set $G^{\prime}:=G \backslash V\left(M_{a b s}\right)$. Using the degree condition, Theorem 1.11 gives us a perfect fractional matching in $G^{\prime}$ for sufficiently large $n$. Lemma 5.6(ii) then transforms this into an almost perfect integer matching $M_{a l m}$ in $G^{\prime}$. We then extend $M_{a l m} \cup M_{a b s}$ to a perfect matching of $G$ by using the absorbing property of $M_{a b s}$. 


\section{An APPLICATION ON THE MINIMUM NUMBER OF DISJOINT CLIQUES IN GRAPHS OF GIVEN EDGE DENSITY}

Another application of Theorem 1.2 concerns the problem of determining the minimum number of disjoint copies of $K_{r}$ in a (2-uniform hyper)graph of given edge density, where $K_{r}$ denotes the complete graph on $r$ vertices. We will use Theorem 1.2 to give a lower bound on this quantity.

Let $b_{r}(m, n)$ be the smallest number of (not necessarily disjoint) copies of $K_{r}$ in a graph with $n$ vertices and $m$ edges. Define

$$
b_{r}(a):=\lim _{n \rightarrow \infty} \frac{b_{r}\left(\left\lfloor a\left(\begin{array}{l}
n \\
2
\end{array}\right)\right\rfloor, n\right)}{\left(\begin{array}{l}
n \\
r
\end{array}\right)} .
$$

Similarly, let $h_{r}(m, n)$ be the smallest number of disjoint copies of $K_{r}$ in a graph with $n$ vertices and $m$ edges. Define

$$
h_{r}(a):=\liminf _{n \rightarrow \infty} \frac{h_{r}\left(\left\lfloor a\left(\begin{array}{l}
n \\
2
\end{array}\right)\right\rfloor, n\right)}{n / r} .
$$

The problem of determining $h_{3}(a)$ was considered by Erdős [7] and Moon [23], but in general very little is known about $h_{r}(a)$. However, $b_{r}(a)$ has been been the focus of much attention and has recently been determined for all $r \geq 3[24,26,27]$. In the interest of deriving a more transparent bound on $h_{r}(a)$, we will also consider a simpler function that bounds $b_{r}(a)$ from below and is quite close to $b_{r}(a)$. Let

$$
b_{r}^{\prime}(a):=\prod_{i=1}^{r-1}(i a-i+1) .
$$

Lovász and Simonovits [20] showed that $b_{r}(a) \geq b_{r}^{\prime}(a)$, for $r \geq 3$ and $a \in[1-1 /(r-1), 1)$.

We now derive our lower bound on $h_{r}(a)$. This bound is unlikely to be close to the correct value of $h_{r}(a)$, but it is better than the bound obtained by greedily removing disjoint copies of $K_{r}$, and for $r \geq 4$ is the only non-trivial result we are aware of.

Corollary 6.1. Let $r \geq 3$. Then

$$
h_{r}(a) \geq \min \left\{r\left(1-\left(1-b_{r}(a)\right)^{1 / r}\right), 0.479\right\} .
$$

In particular, for $a \in[1-1 /(r-1), 1)$,

$$
h_{r}(a) \geq \min \left\{r\left(1-\left(1-\prod_{i=1}^{r-1}(i a-i+1)\right)^{1 / r}\right), 0.479\right\} .
$$

Proof. Let $G=(V, E)$ be a graph with $n$ vertices and $\left\lfloor a\left(\begin{array}{c}n \\ 2\end{array}\right)\right\rfloor$ edges. Then $G$ contains at least $\left(b_{r}(a)+o(1)\right)\left(\begin{array}{l}n \\ r\end{array}\right)$ copies of $K_{r}$. Consider the $r$-uniform hypergraph $H$ with vertex set $V$ and edge set $\left\{e \in\left(\begin{array}{l}V \\ r\end{array}\right): G[e]=K_{r}\right\}$. So $H$ has at least $\left(b_{r}(a)+o(1)\right)\left(\begin{array}{l}n \\ r\end{array}\right)$ edges. So by Theorem 1.2, $H$ has a matching, $M$ say, with

$$
|M| \geq \min \left\{\left(1-\left(1-b_{r}(a)\right)^{1 / r}+o(1)\right) n, 0.479 n / r\right\} .
$$

Then $\{G[e]: e \in M\}$ is a set of $|M|$ disjoint copies of $K_{r}$ in $G$. Hence,

$$
h_{r}(a) \geq \min \left\{r\left(1-\left(1-b_{r}(a)\right)^{1 / r}\right), 0.479\right\} .
$$


Below we plot the edge-density $a$ of a graph (on the horizontal axis) against the lower bound (6.1) on $h_{r}(a)$, for $r=3,4,5$ respectively.
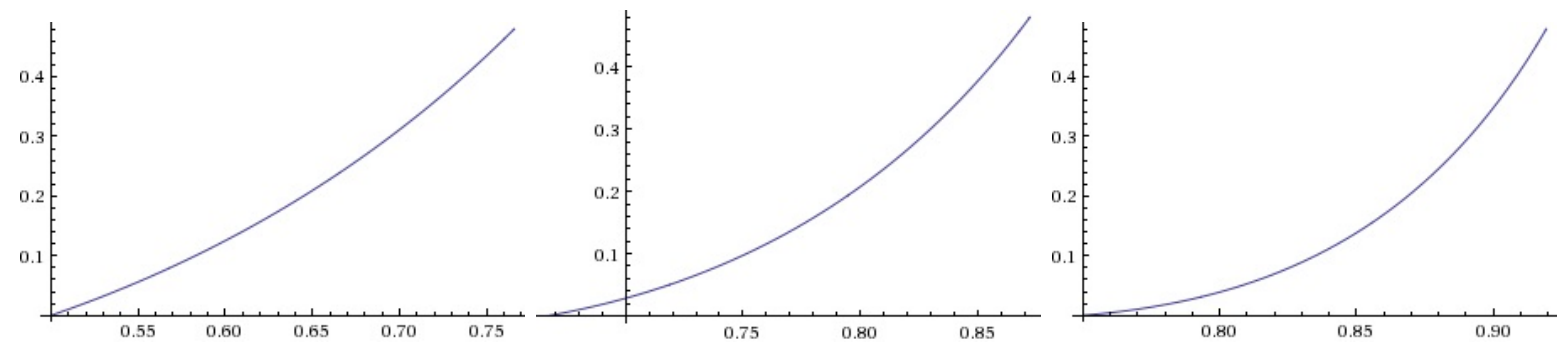

\section{REFERENCES}

[1] N. Alon, P. Frankl, H. Huang, V. Rödl, A. Ruciński, B. Sudakov, Large matchings in uniform hypergraphs and the conjectures of Erdős and Samuels, J. Combin. Theory A 119 (2012), 1200-1215.

[2] N. Alon, H. Huang, B. Sudakov, Nonnegative $k$-sums, fractional covers, and probability of small deviations, J. Combin. Theory B 102 (2012), 784-796.

[3] Z. Baranyai, On the factorization of the complete uniform hypergraph, in: A. Hajnal, R. Rado, V.T. Sós, Infinite and finite sets, Proc. Coll. Keszthely, 1973, Amsterdam, Netherlands: North-Holland, (1975), 91-108.

[4] B. Bollobás, D.E. Daykin, P. Erdős, Sets of independent edges of a hypergraph, Quart. J. Math. Oxford 27 (1976), 25-32.

[5] F.R.K. Chung, Regularity Lemmas for hypergraphs and quasi-randomness, Random Structures and Algorithms 2 (1991), 241-252.

[6] D.E. Daykin, R. Häggkvist, Degrees giving independent edges in a hypergraph, Bull. Austral. Math. Soc. 23 (1981), 103-109.

[7] P. Erdős, Über ein Extremalproblem in der Graphentheorie, Arch. Math. 13 (1962), 222-227.

[8] P. Erdős, A problem on independent r-tuples, Ann. Univ. Sci. Budapest. Eötvős Sect. Math. 8 (1965), 93-95.

[9] P. Erdős, T. Gallai, On maximal paths and circuits of graphs, Acta Math. Acad. Sci. Hung. 10 (1959), 337-356.

[10] P. Erdős, C. Ko, R. Rado, Intersection theorems for systems of finite sets, Quart. J. Math. Oxford 12 (1961), $313-320$

[11] P. Frankl, On the maximum number of edges in a hypergraph with given matching number, arXiv:1205.6847v1 (2012).

[12] P. Frankl, V. Rödl, A. Ruciński, On the maximum number of edges in a triple system not containing a disjoint family of a given size, Combinatorics, Probability and Computing 21 (2012), 141-148.

[13] H. Hàn, Y. Person, M. Schacht, On perfect matchings in uniform hypergraphs with large minimum vertex degree, SIAM J. Discrete Math. 23 (2009), 732-748.

[14] H. Huang, P. Loh, B. Sudakov, The size of a hypergraph and its matching number, Combinatorics, Probability and Computing 21 (2012), 442-450.

[15] I. Khan, Perfect matchings in 3-uniform hypergraphs with large vertex degree, arXiv:1101.5830v3 (2011).

[16] I. Khan, Perfect matchings in 4-uniform hypergraphs, arXiv:1101.5675v2 (2011).

[17] D. Kühn, D. Osthus, Matchings in hypergraphs of large minimum degree, J. Graph Theory 51 (2006), 269-280.

[18] D. Kühn, D. Osthus, Embedding large subgraphs into dense graphs, in: S. Huczynka, J. Mitchell, C. RoneyDougal (Eds.), Surveys in Combinatorics, London Math. Soc. Lecture Note Ser. 365, Cambridge University Press, 2009, 137-167.

[19] D. Kühn, D. Osthus, A. Treglown, Matchings in 3-uniform hypergraphs, J. Combin. Theory B 103 (2013), 291-305.

[20] L. Lovász, M. Simonovits, On the number of complete subgraphs in a graph II, Studies in Pure Math., Birkhäuser, 1983, 459-495.

[21] T. Łuczak, K. Mieczkowska, On Erdős' extremal problem on matchings in hypergraphs, arXiv:1202.4196L (2011). 
[22] K. Markström, A. Ruciński, Perfect matchings and Hamiltonian cycles in hypergraphs with large degrees, European J. Combin. 32 (2011), 677-687.

[23] J.W. Moon, On independent complete subgraphs in a graph, Canad. J. Math. 20 (1968), 95-102.

[24] V. Nikiforov, The number of cliques in graphs of given order and size, Trans. Amer. Math. Soc. 363 (2011), 1599-1618.

[25] O. Pikhurko, Perfect matchings and $K_{4}^{3}$-tilings in hypergraphs of large co-degree, Graphs Combinatorics 24 (2008), 391-404.

[26] A. Razborov, On the minimal density of triangles in graphs, Combinatorics, Probability and Computing $\mathbf{1 7}$ (2008), 603-618.

[27] C. Reiher, Minimizing the number of cliques in graphs of given order and edge density, arXiv:1212.2454v1 (2012)

[28] V. Rödl, A. Ruciński, E. Szemerédi, Perfect matchings in uniform hypergraphs with large minimum degree, European J. Combin. 27 (2006), 1333-1349.

[29] V. Rödl, A. Ruciński, E. Szemerédi, Perfect matchings in large uniform hypergraphs with large minimum collective degree, J. Combin. Theory A 116 (2009), 616-636.

[30] E. Szemerédi, Regular partitions of graphs, Colloq. Internat. CNRS 260 (1978), 399-401.

[31] T. Townsend, PhD Thesis, University of Birmingham, in preparation.

[32] A. Treglown, Y. Zhao, Exact minimum degree thresholds for perfect matchings in uniform hypergraphs II, J. Combin. Theory A, to appear.

School of Mathematics

University of Birmingham

Edgbaston

Birmingham

B15 2TT

$\mathrm{UK}$

E-mail addresses: $\{$ d.kuhn, d.osthus, txt238\}@bham.ac.uk 